\title{
Spatiotemporal pulse shaping with multimode nonlinear guided waves
}

\author{
S. Wabnitz ${ }^{1,2}$, K. Krupa ${ }^{1}$, D. Modotto ${ }^{1}$, G. Millot ${ }^{3}$, D. S. Kharenko ${ }^{2,4}$, V. A. Gonta ${ }^{2}$, E.V. Podivilov ${ }^{2,4}$, S. Babin ${ }^{2,4}$, \\ A.Tonello ${ }^{5}$, A. Barthélémy ${ }^{5}$ and V. Couderc ${ }^{5}$ \\ ${ }^{1}$ Dipartimento di Ingegneria dell'Informazione, Università di Brescia, and INO-CNR, Brescia, Italy \\ ${ }^{2}$ Novosibirsk State University, 1 Pirogova str., Novosibirsk 630090, Russia \\ ${ }^{3}$ Université de Bourgogne Franche-Comté, ICB, UMR CNRS 6303, Dijon, France \\ ${ }^{4}$ Institute of Automation and Electrometry, SB RAS, 1 ac. Koptyug ave., Novosibirsk 630090, Russia \\ ${ }^{5}$ Université de Limoges, XLIM, UMR CNRS 7252, Limoges, France \\ stefan.wabnitz@unibs.it
}

\begin{abstract}
We experimentally and theoretically investigate complex temporal pulse reshaping that accompanies Kerr beam self-cleaning in multimode optical fibers. We also study the output beam shape dependence on initial conditions.
\end{abstract}

Keywords-Pulse propagation and optical solitons; transverse effects; nonlinear fiber optics; fiber lasers.

Nonlinear multimode optical fibers (MMFs) have recently emerged as easily accessible platform to control complex spatiotemporal beam reshaping phenomena [1]. Light intensity oscillations associated to the self-imaging effect in gradedindex (GRIN) MMFs lead, via the Kerr effect, to a dynamic long-period index grating which may phase-match the generation of ultra-broadband sideband series [2-4]. For relatively short, virtually lossless GRIN fibers, beam selfcleaning activated by the Kerr effect is observed, at lower power thresholds than the Raman beam cleanup $[5,6]$. The output highly multimode speckled beam evolves, at high powers, into a high brightness bell-shaped beam sitting on a low-power background of high-order modes. This Kerr beam self-cleaning is shown to be even reinforced in the presence of strong loss or gain, e.g., in a passive or active ytterbium doped MMF [7], which leads to its possible exploitation in high power multimode fiber laser sources [8].

We shall overview recent experiments, which demonstrate the spatiotemporal pulse break-up and significant temporal compression that accompany the self-cleaning process [9]. At the same time, we shall describe experiments revealing the dependence of the output beam shape and the efficiency of the self-cleaning process on the input beam conditions, such as transverse dimension and incidence angle [10].

Although numerical simulations based on the full 3D nonlinear Schroedinger equation with a parablic potential reproduce well the beam cleaning effects in GRIN fibers [5], to gain a physical understanding of its underlying modal redistribution it is necessary to resort to (approximate) modal developments of the propagation equation. We shall present the results of such

This work was sponsored by the Ministry of Education and Science of the Russian Federation (Minobrnauka) (14.Y26.31.0017); Horiba Medical and BPI france within the Dat@diag project; iX- core research foundation; French National Research Agency ANR Labex ACTION; the European Union's Horizon 2020 research and innovation program under the Marie Skl odowska- Curie grant agreement No. 2015-713694 . studies, suggesting that the origin of the observed nonlinear modal reshaping is a new form of parametric instability.

\section{REFERENCES}

[1] L.G. Wright, D.N. Christodoulides, and F.W. Wise, "Controllable spatiotemporal nonlinear effects in multimode fibres," Nature Photonics, Vol. 9, pp. 306-310, 2015

[2] L.G. Wright, S. Wabnitz, D.N. Chistodoulides, and F.W. Wise, "Ultrabroadband dispersive radiation by spatiotemporal oscillation of multimode waves," Phys. Rev. Lett., Vol. 115, 223902, 2015.

[3] K. Krupa, A. Tonello, A. Barthélémy, V. Couderc, B. Mohamed Shalaby, A. Bendahmane, G. Millot, and S. Wabnitz, "Observation of geometric parametric instability induced by the periodic spatial selfimaging of multimode waves," Phys. Rev. Lett., 116, 183901 (2016).

[4] L.G. Wright, Z. Liu, D.A. Nolan, M.-J. Li, D.N. Christodoulides, and F.W. Wise, "Self-organized instability in graded-index multimode fibres," Nature Photonics 10, 771-776 (2016).

[5] K. Krupa, A. Tonello, B.M. Shalaby, A. Barthélémy, G. Millot, S. Wabnitz, and V. Couderc, "Spatial beam self-cleaning in multimode fibres," Nat. Photonics 11, 237-241 (2017).

[6] Z. Liu, L.G. Wright, D.N. Christodoulides, and F.W. Wise, "Kerr selfcleaning of femtosecond-pulsed beams in graded-index multimode fiber," Opt. Lett. 41, 3675-3678 (2016).

[7] R. Guenard, K. Krupa, R. Dupiol, M. Fabert, A. Bendahmane, V. Kermene, A. Desfarges-Berthelemot, J.L. Auguste, A. Tonello, A. Barthélémy, G. Millot, S. Wabnitz, and V. Couderc, "Kerr self-cleaning of pulsed beam in an ytterbium doped multimode fiber," Optics Express 25, 4783-4792 (2017).

[8] R. Guenard, K. Krupa, R. Dupiol, M. Fabert, A. Bendahmane, V. Kermene, A. Desfarges-Berthelemot, J.L. Auguste, A. Tonello, A. Barthélémy, G. Millot, S. Wabnitz, and V. Couderc, "Nonlinear beam self-cleaning in a coupled cavity composite laser based on multimode fiber," Opt. Express 25, 22219-22227 (2017).

[9] K. Krupa, A. Tonello, V. Couderc, A. Barthélémy, G. Millot, D. Modotto, and S.Wabnitz, "Spatiotemporal light beam compression from complex nonlinear mode mixing," arXiv:1711.11478 [physics.optics] (2017).

[10] S.Wabnitz, D. S. Kharenko, V. A. Gonta, K. Krupa, E. V. Podivilov, S. A. Babin, "Spatial beam self-cleaning conditions in multimode GRIN fibers with different core diameter," in preparation (2018). 